\title{
O instrumento de avaliação de qualidade de vida da Organização Mundial da Saúde (WHOQOL-100): características e perspectivas
}

\author{
The World Health Organization instrument \\ to evaluate quality of life (WHOQOL-100): \\ characteristics and perspectives
}

Marcelo Pio de Almeida Fleck 1

\begin{abstract}
Quality of life evaluation has been growing in importance as a measure to evaluate results in medicine. Taking into account the absence of a quality of life instrument with a cross-cultural approach, World Health Organization developed a unique methodology to create it. Initially, the World Health Organization Quality of Life instrument (WHOQOL-100), with a hundred questions, was elaborated. The need of a short instrument to be used in extensive epidemiological studies caused WHO to develop a short 26-question version (WHOQOLbref). Nowadays, a specific module to evaluate quality of life in HIV/Aids patients and a module to evaluate spirituality, religiousness and personal beliefs are being developed.
\end{abstract}

Key words Quality of Life; World Health Organization; Rating Scale
Resumo A avaliação de qualidade de vida vem crescendo em importância como medida de avaliação de resultados de tratamento em medicina. A partir da constatação da falta de um instrumento de avaliação de qualidade de vida com um enfoque transcultural, a OMS desenvolveu uma metodologia única para sua criação. Inicialmente foi desenvolvido o World Health Organization Quality of Life (WHOQOL-100), instrumento composto de cem questões. A necessidade de um instrumento mais curto para uso em extensos estudos epidemiológicos fez com que a OMS desenvolvesse a versão abreviada com 26 questões (o WHOQOL-Bref). Atualmente, estão em desenvolvimento dois módulos: um específico para avaliar qualidade de vida em pacientes com HIV/Aids e outro para avaliar espiritualidade, religiosidade e crenças pessoais.

Palavras-chave Qualidade de vida; Organização Mundial da Saúde; Escala de Avaliação

1 Departamento de 
A Organização Mundial da Saúde (OMS) definiu saúde como um completo estado de bemestar físico, mental e social e não meramente a ausência de doença (WHO, 1946). No entanto, as políticas em saúde e a própria formação dos profissionais sempre colocaram a prioridade no controle da morbidade e mortalidade. Apenas recentemente vem havendo uma crescente preocupação não só com a freqüência e a severidade das doenças, mas também com a avaliação de medidas de impacto da doença e comprometimento das atividades diárias (Bergner et al., 1981), medidas de percepção da saúde (Hunt et al., 1985) e medida de disfunção/status funcional (Ware et al. 1992).

Não há dúvida de que o desenvolvimento de instrumentos e formas de avaliação de mortalidade e morbidade é uma tarefa muito mais simples (ou muito menos complexa) do que criar instrumentos para avaliar qualidade de vida ou bem-estar. É difícil definir construtos subjetivos influenciados por características temporais (de época) e culturais, como estes em questão. Assim, desenvolver instrumentos para avaliar qualidade de vida psicometricamente válidos é um grande desafio. Some-se a isto o fato de que a maioria desses instrumentos foi desenvolvida nos Estados Unidos e na Europa, o que torna o seu uso transcultural no mínimo questionável.

A ausência de um instrumento que avaliasse qualidade de vida per se, com uma perspectiva internacional, fez com que a OMS constituísse um Grupo de Qualidade de Vida (Grupo WHOQOL) com a finalidade de desenvolver instrumentos capazes de fazê-lo dentro de um perspectiva transcultural.

\section{Aspectos conceituais}

Como não há um consenso sobre a definição de qualidade de vida, o primeiro passo para o desenvolvimento do instrumento World Health Organization Quality of Life (WHOQOL) foi a busca da definição do conceito. Assim, a OMS reuniu especialistas de várias partes do mundo, que definiram qualidade de vida como $a$ percepção do indivíduo de sua posição na vida no contexto da cultura e sistema de valores nos quais ele vive e em relação aos seus objetivos, expectativas, padrões e preocupações (The WHOQOL Group, 1995). É um conceito amplo que abrange a complexidade do construto e inter-relaciona o meio ambiente com aspec- tos físicos, psicológicos, nível de independência, relações sociais e crenças pessoais.

A definição do Grupo WHOQOL reflete a natureza subjetiva da avaliação que está imersa no contexto cultural, social e de meio ambiente. O que está em questão não é a natureza objetiva do meio ambiente, do estado funcional ou do estado psicológico, ou ainda como o profissional de saúde ou um familiar avalia essas dimensões: é a percepção do respondente/paciente que está sendo avaliada.

\section{O método WHOQOL}

O WHOQOL foi desenvolvido utilizando um enfoque transcultural original. Primeiro, por envolver a criação de um único instrumento de forma colaborativa simultaneamente em diferentes centros. Desta forma, vários centros com culturas diversas participaram da operacionalização dos domínios de avaliação de qualidade de vida, da redação e seleção de questões, da derivação da escala de respostas e do teste de campo nos países envolvidos nesta etapa. Com esta abordagem foi possível equacionar as dificuldades referentes à padronização, equivalência e tradução à medida que se desenvolvia o instrumento. Para garantir que a colaboração fosse genuinamente internacional, os centros foram selecionados de forma a incluir países com diferenças no nível de industrialização, disponibilidade de serviços de saúde, importância da família e religião dominante, entre outros.

Segundo, o método WHOQOL utilizou uma entrada de dados iterativa entre os pesquisadores com a consolidação e revisão da informação em cada estágio do desenvolvimento do instrumento. Isso permitiu que as impressões dos especialistas em qualidade de vida, bem como a visão dos pacientes e profissionais de saúde estivessem contínua e repetidamente influenciando o processo.

Terceiro, um cuidadoso método de tradução do instrumento - que envolveu não só a tradução e retrotradução, mas também a discussão em grupos focais da versão com pacientes, profissionais de saúde e membros da comunidade - permitiu a incorporação de várias sugestões às traduções.

O método WHOQOL aplicado à versão brasileira do instrumento foi descrito detalhadamente em outra publicação (Fleck et al., 1999a). 


\section{Os instrumentos}

\section{WHOQOL-100}

O instrumento WHOQOL-100 consiste em cem perguntas referentes a seis domínios: físico, psicológico, nível de independência, relações sociais, meio ambiente e espiritualidade/religiosidade/crenças pessoais. Esses domínios são divididos em 24 facetas (Quadro 1). Cada faceta é composta por quatro perguntas. Além das 24 facetas específicas, o instrumento tem uma 25a composta de perguntas gerais sobre qualidade de vida.

As respostas para as questões do WHOQOL são dadas em uma escala do tipo Likert. As perguntas são respondidas através de quatro tipos de escalas (dependendo do conteúdo da pergunta): intensidade, capacidade, freqüência e avaliação. Utilizou-se uma cuidadosa metodologia para selecionar as palavras que compõem as escalas em cada idioma (Szabo et al., 1997), com a finalidade de manter a equivalência nas diferentes línguas (Fleck et al., 1999a).

As características psicométricas do WHOQOL-100 foram estabelecidas a partir de uma amostra de 8.294 indivíduos provenientes de 19 centros (The WHOQOL Group, 1998a). Avaliou-se a consistência interna do instrumento utilizando o coeficiente de Cronbach. A validade discriminante para os itens foi determinada através do teste $t$ para distinguir entre controles e doentes. A confiabilidade teste-reteste foi avaliada por meio do coeficiente de correlação de Pearson $(r)$. A contribuição dos escores dos domínios foi avaliada utilizando regressão múltipla. As características psicométricas foram bastante boas. O instrumento apresenta boa consistência interna, boa validade discriminante para doentes e normais. $\mathrm{O}$ instrumento permaneceu estável em duas medidas sucessivas (confiabilidade teste-reteste). Todos os domínios contribuíram de forma significativa para explicar a variância observada na faceta geral de qualidade de vida e saúde geral, com o meio ambiente contribuindo mais, e o domínio da espiritualidade, menos (Harper \& Power, 1998). As características psicométricas da versão em português foram semelhantes (Fleck et al., 1999b), com a diferença de que o domínio VI (espiritualidade/religião/crenças pessoais) não apresentou capacidade estatisticamente significativa de discriminar entre controles e doentes e o mes-

\section{Quadro 1}

Domínios e facetas do WHOQOL.

\section{Domínio I - domínio físico}

1. dor e desconforto

2. energia e fadiga

3. sono e repouso

Domínio II - domínio psicológico

4. sentimentos positivos

5. pensar, aprender, memória e concentração

6. auto-estima

7. imagem corporal e aparência

8. sentimentos negativos

\section{Domínio III - nível de independência}

9. mobilidade

10. atividades da vida cotidiana

11. dependência de medicação ou de tratamentos

12. capacidade de trabalho

\section{Domínio IV - relações sociais}

13. relações pessoais

14. suporte (apoio) social

15. atividade sexual

\section{Domínio V - meio ambiente}

16. segurança física e proteção

17. ambiente no lar

18. recursos financeiros

19. cuidados de saúde e sociais: disponibilidade e qualidade

20. oportunidades de adquirir novas informações e habilidades

21. participação em, e oportunidades de recreação/lazer

22. ambiente físico: (poluição/ruído/trânsito/clima)

23. transporte

Domínio VI - aspectos espirituais/religião/

crenças pessoais

24. espiritualidade/religiosidade/crenças pessoais

mo domínio não contribuiu de forma significativa para explicar a variância observada na faceta geral.

\section{O WHOQOL-bref}

Ao proporcionar uma avaliação detalhada das 24 facetas que o compõem, o WHOQOL pode tornar-se muito extenso para algumas aplicações; por exemplo, em grandes estudos 
epidemiológicos em que a avaliação de qualidade de vida é apenas uma das variáveis em estudo. A necessidade de um instrumento mais curto, que demandasse pouco tempo para o preenchimento e que preservasse características psicométricas satisfatórias, fez com que o Grupo de Qualidade de Vida da OMS desenvolvesse uma versão abreviada do WHOQOL100, o WHOQOL-bref.

O WHOQOL-bref consta de 26 questões. Duas questões são gerais de qualidade de vi$\mathrm{da}$, ao passo que as demais representam cada uma das 24 facetas que compõem o instrumento original (Quadro 2) (The WHOQOL Group, 1998b).

Diferente do WHOQOL-100 em que cada uma das 24 facetas é avaliada a partir de qua-

Quadro 2

Domínios e facetas do WHOQOL-bref.

\section{Domínio I - domínio físico}

1. dor e desconforto

2. energia e fadiga

3. sono e repouso

10. atividades da vida cotidiana

11. dependência de medicação ou de tratamentos

12. capacidade de trabalho

\section{Domínio II - domínio psicológico}

4. sentimentos positivos

5. pensar, aprender, memória e concentração

6. auto-estima

7. imagem corporal e aparência

8. sentimentos negativos

24. espiritualidade/religiosidade/crenças pessoais

Domínio III - relações sociais

13. relações pessoais

14. suporte (apoio) social

15. atividade sexual

\section{Domínio IV - meio ambiente}

16. segurança física e proteção

17. ambiente no lar

18. recursos financeiros

19. cuidados de saúde e sociais: disponibilidade e qualidade

20. oportunidades de adquirir novas informações e habilidades

21. participação em, e oportunidades de recreação/lazer

22. ambiente físico: (poluição/ruído/trânsito/clima)

23. transporte tro questões, no WHOQOL-bref cada faceta é avaliada por apenas uma questão. Os dados que deram origem à versão abreviada foram extraídos do teste de campo de 20 centros em 18 países (The WHOQOL Group, 1998b).

O critério de seleção das questões para compor a versão abreviada foi tanto psicométrico como conceitual. No nível conceitual, o Grupo de Qualidade de Vida da OMS definiu que o caráter abrangente do instrumento original deveria ser preservado. Assim, cada uma das 24 facetas que compõem o WHOQOL-100 deveria ser representada por uma questão. No nível psicométrico, selecionou-se, então, a questão que mais altamente se correlacionasse ao escore total do WHOQOL-100, calculado pela média de todas as facetas. Depois dessa etapa, um grupo de especialistas examinou os itens selecionados para estabelecer se representavam conceitualmente o domínio de origem das facetas. Dos 24 itens selecionados, seis foram substituídos por questões que melhor definissem a faceta correspondente. Três do domínio Meio Ambiente foram substituídos por serem muito correlacionados com o domínio Psicológico. Os outros três outros itens foram substituídos por explicarem melhor a faceta em questão.

Realizou-se uma análise fatorial confirmatória para uma solução a quatro domínios. O WHOQOL-bref, então, passou a ser composto pelos domínios: físico, psicológico, relações sociais e meio ambiente.

Um teste de campo com o WHOQOL-bref foi aplicado em vários centros com a finalidade de avaliar suas características psicométricas, e os resultados devem ser publicados em breve. A versão em português do instrumento apresentou características satisfatórias de consistência interna, validade discriminante, validade de critério, validade concorrente e fidedignidade teste-reteste (Fleck et al., 2000, no prelo).

\section{Desenvolvimentos futuros}

\section{O módulo HIV/Aids}

O WHOQOL foi desenvolvido partindo do princípio de que o conceito de qualidade de vida é abrangente e pode ser aplicado a várias doenças e a situações não-médicas. No entanto, para alguns grupos de pessoas ou de doenças, o WHOQOL pode não avaliar qualidade 
de vida de forma suficientemente completa ou apropriada. Nesses casos, estaria indicado o desenvolvimento de um módulo do WHOQOL que, ao ser usado em combinação com o instrumento nuclear (WHOQOL-100), avaliaria as circunstâncias e peculiaridades da doença em questão (WHO, 1993). A OMS identificou cinco áreas prioritárias para o desenvolvimento de módulos: pessoas sofrendo de doenças crônicas (epilepsia, artrite, câncer, diabetes); pessoas que cuidam de pessoas doentes ou com limitações decorrentes da doença (quem cuida de pacientes terminais); pessoas vivendo em situações de estresse intenso (campos de refugiados); pessoas com dificuldade de comunicação (com distúrbios severo de aprendizado) e crianças (WHO, 1993).

O primeiro módulo do WHOQOL a ser desenvolvido foi o de HIV/Aids, em função da importância médica da doença, do impacto na qualidade de vida, de seu estigma e das peculiaridades que envolvem o contágio.

Inicialmente, reuniram-se especialistas representando oito países dos diferentes continentes (incluindo os países com grande número de casos como Brasil, os Estados Unidos, Zâmbia, Zimbabwe e Índia). Várias questões específicas foram levantadas sobre as peculiaridades que os pacientes com HIV/Aids possuíam no que tange à sua avaliação de qualidade de vida. Como exemplo, foram consideradas a rejeição social, a sensação de sobrecarga do sistema de apoio social, a culpa por essa sobrecarga, o impacto na sexualidade, o medo de infectar os outros além de vários outros aspectos.

Para desenvolver o módulo HIV/Aids, o grupo WHOQOL utilizou uma metodologia semelhante à empregada para o desenvolvimento do instrumento original, o WHOQOL100. O trabalho foi dividido em várias etapas (WHO, 1997):

1) discussão do instrumento original (WHOQOL-100) e avaliação das limitações para pessoas com HIV/Aids;

2) discussão em grupos focais preliminares com pessoas soropositivas assintomáticas, sintomáticas e com Aids, para avaliar a necessidade de facetas adicionais;

3) discussão em grupos focais sobre as possíveis novas facetas com pessoas soropositivas assintomáticas, sintomáticas, com Aids e com familiares e cuidadores;

4) painel em cada centro para elaboração de facetas adicionais;
5) tradução para o inglês;

6) desenvolvimento de um instrumentopiloto;

7) tradução do piloto segundo a metodologia da OMS (Sartorius \& Kuyken, 1994; Fleck et al., 1999a);

8) teste de campo inicial em seis países (Brasil, Zâmbia, Zimbabwe, Índia, Austrália e Tailândia);

9) análise de resultados e redação do Módulo HIV/Aids.

O teste de campo foi realizado, estando os dados em processo de análise.

\section{Módulo espiritualidade, religiosidade e crenças pessoais}

Nos últimos anos a preocupação e a valorização da dimensão 'não-material' ou espiritual em saúde tem crescido em importância (Ellerhorst-Ryan, 1996). Uma resolução da 101ạ sessão da Assembléia Mundial de Saúde propôs uma modificação do conceito de saúde da OMS para um estado dinâmico de completo bem-estar físico, mental, espiritual e social.

No instrumento WHOQOL-100, existe um domínio denominado Espiritualidade, Religiosidade e Crenças pessoais (domínio VI). No entanto, esse domínio consta de apenas uma faceta (ver Quadro 1). O objetivo deste recente projeto do Grupo WHOQOL é aprofundar esse domínio, examinando, em diferentes culturas e religiões, as facetas que o integram. Assim, talvez seja possível e interessante desenvolver um módulo a partir da medida já existente no WHOQOL e torná-la uma medida mais sensível e completa desse domínio para utilização na área de saúde. Neste caso, entraria como um módulo adicional ao WHOQOL para ser usado quando necessário. É importante salientar que este módulo não é dirigido a qualquer religião específica, mas a todas as formas de espiritualidade, praticada ou não através de religiões formais. Para os que não são afiliados a religião alguma ou dimensão espiritual, o domínio deve referir-se a crenças ou códigos de comportamento (WHO, 1998).

A metodologia a ser utilizada é semelhante àquela descrita para o módulo de HIV/Aids. Serão realizados grupos focais com pacientes com doenças agudas, crônicas e terminais, profissionais de saúde, membros das principais crenças religiosas de cada país envolvido, 
além de grupos de ateus e com crenças outras que não religiosas (movimento ecológico, por exemplo).

Este projeto começou no ano de 1999, estando envolvidos em torno de vinte países, incluindo o Brasil.

\section{Conclusão}

O Grupo WHOQOL tem trabalhado para desenvolver medidas que avaliem a qualidade de vida dentro de uma perspectiva internacional em que os diferentes países e culturas possam influenciar desde a elaboração dos conceitos que norteiam a elaboração das questões que farão parte do instrumento até sua validação.

As aplicações desses instrumentos são amplas e incluem não somente a prática clínica individual, mas também a avaliação de efetividade de tratamentos e de funcionamento de serviços de saúde. Além disso, podem ser importantes guias para políticas de saúde.

Sem dúvida, existem inúmeras dificuldades conceituais e de desenvolvimento de questões importantes do ponto de vista psicométrico. No entanto, este desafio precisa ser enfrentado, já que vida é complexidade e não há razão para não se aplicar um esforço semelhante para avaliar qualidade de vida do que é feito para avaliar Neuroimagem. (Katschnig \& Angermeyer, 1997).

\section{Referências bibliográficas}

Bergner R et al. 1981. The sickness impact profile: development and final revision of a health status measure. Medical Care 19:787-805.

Ellerhorst-Ryan JM 1996. Instruments to measure spiritual status, pp. 145-153. In B Spilker (ed.). Quality of Life and Pharmacoeconomics in Clinical Trials. Lippincott-Raven, Filadélfia.

Fleck MPA et al. 1999a. Desenvolvimento da versão em português do instrumento de avaliação de qualidade de vida da OMS (WHOQOL-100). Revista Brasileira de Psiquiatria 21(1):19-28.

Fleck MPA et al. 1999b. Aplicação da versão em português do instrumento de avaliação de qualidade de vida da OMS (WHOQOL-100). Revista de Saúde Pública 33(2):198-205.

Fleck MPA et al. 2000. O instrumento de avaliação de qualidade de vida abreviado da Organização Mundial da Saúde (WHOQOL-breve): aplicação da versão em português. Revista de Saúde Pública 22(2), (no prelo).

Harper A \& Power M 1998. On behalf of the WHOQOL Group: WHOQOL user manual (Draft). OMS, Genebra, $88 \mathrm{pp}$.

Hunt SM, Mcewen J \& Mckenna SP 1985. Measuring health status. Journal of the Royal College of General Practioner 35:185-188.

Katschnig H \& Angermeyer M 1997. Quality of life in depression, pp. 137-147. In H Katschnig, H Freeman \& Sartorius N. Quality of Life in Mental Disorders. Wiley, Chichester.

Sartorius N \& Kuyken W 1994. Translation of health status instruments, pp. 3-18. In JE Orley \& W Kuiken (eds.). Quality of Life Assessment: International Perspectives. Springer Verlag, Berlim.

Szabo S et al. 1997. An approach to response scale development for cross-cultural questionnaires. European Psychologist 2(3):270-276.

Ware JE, Sherbourne CD 1992. The MOS 36 item Short Form Health Status Survey (SF-36):1. Conceptual framework and item selection. Medical Care 30(6):473-483.

The WHOQOL Group 1995. The World Health Organization quality of life assessment (WHOQOL): position paper from the World Health Organization. Social Science and Medicine 10:1403-1409.

The WHOQOL Group 1998a. The World Health Organization quality of life assessment (WHOQOL): development and general psychometric properties. Social Science and Medicine 12:1569-1585.

The WHOQOL Group 1998b. Development of the World Health Organization WHOQOL-B: quality of life assessment. Psychological Medicine 28:551-558.

WHO (World Health Organization) 1946. Constitution of the World Health Organization. Basic Documents. WHO. Genebra.

WHO (World Health Organization) 1993. WHOQOL: study protocol. MNH/PSF/93.9. WHO, Genebra. 39 pp.

WHO (World Health Organization) 1997. Country protocol for developing the WHO quality of life (WHOQOL): HIV/Aids module. MNH/PSF/97.3. WHO, Genebra. 18 pp.

WHO (World Health Organization) 1998. WHOQOL and spirituality, religiousness and personal beliefs (SRPB). Report on WHO consultation. MNH/MAS/ MHP/98.2 WHO, Genebra. 22 pp.

OBS: Estas três últimas referências são publicações da Organização Mundial da Saúde e são distribuídas pela OMS a partir de solicitação. O código $\mathrm{MNH} /$ MAS/MHP refere-se ao setor de origem do documento seguido pelo ano e número. Têm sido citadas na literatura desta forma (ver The WHOQOL Group 1995. The World Health Organization quality of life assessment (WHOQOL): position paper from the World Health Organization. Social Science and Medicine 10:1403-1409). 